\title{
PARÂMETROS E TENDÊNCIAS GENÉTICAS PARA CARACTERÍSTICAS PRODUTIVAS DE BOVINOS DA RAÇA NELORE
}

\author{
GENETIC PARAMETER AND TREND FOR PRODUCTIVE TRAITS OF \\ NELLORE CATTLE
}

\author{
Julio César de Souza1* \\ Fabrício José Parisi da Fonseca² \\ Luiz Otávio Campos da Silva ${ }^{3}$ \\ Rosana Moreira da Silva de Arruda ${ }^{1}$ \\ Andrea Gondo ${ }^{3}$ \\ José Antonio de Freitas ${ }^{4}$ \\ 1 Universidade Federal de Mato Grosso do Sul, Aquidauana, MS, Brasil. \\ 2Universidade Estadual de Mato Grosso do Sul, Aquidauana, MS, Brasil. \\ ${ }^{3}$ Centro Nacional em Pesquisa de Gado de Corte - EMBRAPA, Campo Grande, MS, Brasil. \\ ${ }^{4}$ Universidade Federal do Paraná, Palotina, PR, Brasil. \\ *Autor para correspondência - julio.souza@ufms.br
}

\section{Resumo}

A região Centro-Oeste tem se destacado como produtora de carne bovina e, para tanto, produzir com eficiência tem sido a meta dos produtores. Nesse sentido, objetivou-se estimar parâmetros e tendências genéticas para pesos aos 240 (P240) e 420 (P420) dias de idade em bovinos da raça Nelore, criados na região de Goiás, Brasil. Utilizaram-se 48.580 dados para P240 e 28.685 para P420. Os parâmetros genéticos foram obtidos por análises univariadas em que o modelo continha os efeitos fixos de grupo de contemporâneos (estação de nascimento - águas e seca -, ano de nascimento, sexo e fazenda) e a covariável idade da vaca. Como efeito aleatório, utilizou-se o efeito aditivo direto e materno para P240 e apenas direto para P420. As tendências genéticas foram obtidas por meio da análise de regressão do valor genético do animal sobre ano de nascimento. Os resultados obtidos de herdabilidade direta foram $0,15 \pm 0,02$ para P240 e 0,24 $\pm 0,00$ para P420. O ganho genético anual foi de $0,274 \mathrm{~kg}$ para P240 e de $0,506 \mathrm{~kg}$ P420. Os resultados indicam a possibilidade de se obterem maiores ganhos genéticos por meio da seleção, que está sendo mais eficiente sobre o P420.

Palavras-chave: ganho genético; herdabilidade; peso.

\begin{abstract}
The Midwest region has excelled as a beef producer and producing efficiently has been the goal of livestock farmers. This study aimed to estimate the genetic parameters and trends for weights at 240(W240) and 420(W420) days of age in Nellore cattle, reared in Goias state, Brazil. A total of 48,580 data for W240 and 28,685 for W420 were used. Genetic parameters were obtained by univariate analysis. The model included the fixed effects of contemporary group (birth season-dry and wet-, year of birth, sex and farm) and covariate age of cow. Direct and maternal effects were used as random effect for W240 and the direct effect for W420. The genetic trends were obtained by regression analysis of the genetic value of the animal on the year of birth. The results of direct
\end{abstract}


heritability were $0.15 \pm 0.02$ for W240 and $0.24 \pm 0.00$ for W420. The annual genetic gain was 0.311 $\mathrm{kg}$ for W240 and $0.511 \mathrm{~kg}$ for 420 days of age (W420). The results indicate the possibility of obtaining higher genetic gains through selection; however, the selection is being more efficient at W420.

Keywords: genetic gain; heritability; weight.

Recebido em: 08 de julho de 2013

Aceito em 15 de março de 2018

\section{Introdução}

Os programas de melhoramento genético favorecem diretamente a posição de destaque do Brasil no mercado internacional da pecuária bovina. Esses programas utilizam, principalmente, as características de crescimento dos animais como critérios de seleção, devido à sua fácil obtenção e às herdabilidades de magnitude média a alta, gerando animais que apresentam maiores ganhos de peso em determinadas idades $^{(1)}$.

Peso aos 240 dias (P240) e peso aos 420 dias (P420) assumem uma grande importância em programas de seleção para melhoramento genético de bovinos de corte. Tal importância se dá pelo fato de que, através do P240, pode-se fazer uma estimativa das habilidades maternas da matriz (produção de leite, assistência ao bezerro) podendo observar as qualidades das matrizes como mães e quais estão ofertando bezerros mais pesados ao desmame. Com relação ao P420, pode-se ter uma estimativa do potencial genético do próprio animal, também considerando que já é uma idade mais próxima ao destino final do animal, que na pecuária de corte é o abate.

As estimativas de parâmetros genéticos para características ponderais na raça Nelore têm apresentado grande variabilidade, sendo um bom indicador da resposta à seleção ou melhoramento genético a ser alcançado $^{(2)}$. Por meio do conhecimento de parâmetros genéticos e de estimativas de mudança genética, é possível realizar o acompanhamento e estabelecimento de diretrizes que guiem os programas de melhoramento genético, avaliando o progresso genético ao longo do tempo para que os resultados sirvam de elementos orientadores para ações futuras ${ }^{(3)}$.

Neste contexto, objetivou-se estimar os parâmetros e as tendências genéticas para peso aos 240 e 420 dias de idade em bovinos da raça Nelore, criados na região de Goiás.

\section{Material e métodos}

Foram analisadas as informações de animais da raça Nelore criados na região de Goiás, região $30^{(4)}$, entre os anos de 1976 a 2003. Os dados são pertencentes ao Arquivo Zootécnico Nacional - Raças Zebuínas (Convênio de Associação Brasileira dos Criadores de Zebu com a Empresa Brasileira de Pesquisa Agropecuária - ABCZ/EMBRAPA). Os pesos foram padronizados aos 240 (P240) e 420 (P420) dias de idade utilizando as seguintes fórmulas: 


$$
\begin{gathered}
\mathrm{P} 240=\left(\frac{\mathrm{Pdesm}-\mathrm{PN}}{\text { idade }}\right) * 240+\mathrm{PN} \\
\mathrm{P} 420=\left(\frac{\mathrm{P} 450-\mathrm{P} 365}{\text { dif_idade }}\right) * 180+\mathrm{P} 240
\end{gathered}
$$

Em que: P240 e P420 são os pesos ajustados as respectivas idades; 180 e 240 são constantes de ajustes; PN é o peso ao nascer; P450 e P365 são pesos observados em campo; idade representa a idade da pesagem ao desmame; dif_idade corresponde à diferença entre essas duas idades.

O modelo estatístico continha os efeitos aleatórios aditivo direto, aditivo materno para P240 e apenas aditivo direto para $\mathrm{P} 420$. O efeito fixo de grupo de contemporâneos, formados por animais nascidos no mesmo ano e época (seca = maio a setembro, e águas = outubro a abril), do mesmo sexo e mantidos na mesma fazenda, além da (co)variável idade da vaca ao parto (efeito linear e quadrático). Os grupos contemporâneos formados tinham, no mínimo, 10 indivíduos e touros com mínimo cinco filhos, na tabela 1 podemos encontrar os números de observações de acordo com a variável analisada.

Tabela 1. Números de observações de acordo com a variável analisada

\begin{tabular}{lll}
\hline & P240 & P420 \\
\hline Grupo contemporâneo & 5.606 & 4.166 \\
$\mathrm{~A}^{-1}$ & 88.673 & 81.673 \\
$\mathrm{~N}^{\circ}$. animais & 48.580 & 28.685 \\
\hline
\end{tabular}

$\mathrm{A}^{-1}:$ Número de informações na matriz de parentesco.

Para obter as estimativas dos parâmetros genéticos, empregou-se o modelo animal, utilizando o aplicativo Multiple Traits Derivate Free Restrict Maximum Likelihood, MTDFREML (https://aipl.arsusda.gov/software/mtdfreml/). Foram utilizados dois modelos matriciais:

$$
\begin{array}{ll}
\mathrm{y}=\mathrm{X} \beta+\mathrm{Zg}+\mathrm{Mm}+\mathrm{Wp}+\mathrm{e} & {[\mathrm{P} 240]} \\
\mathrm{y}=\mathrm{X} \beta+\mathrm{Zg}+\mathrm{Wp}+\mathrm{e} & {[\mathrm{P} 420]}
\end{array}
$$

Em que: $y$ corresponde ao vetor de observações; $X$ representa a matriz de incidência dos efeitos fixos; $\beta$ é o vetor de efeitos fixos; $Z$ representa a matriz de incidência dos efeitos genéticos aditivos diretos; $g$ corresponde ao vetor dos efeitos genéticos aditivos diretos; $M$ é a matriz de incidência dos efeitos genéticos aditivos maternos; $m$ simboliza o vetor de efeitos genéticos aditivos maternos; $W$ representa a matriz de incidência dos efeitos de ambiente materno permanente; $p$ é o vetor dos efeitos de ambiente materno permanente; $e$ corresponde ao erro residual.

O critério de convergência adotado foi $10^{-6}$. A cada convergência, o programa era reiniciado, utilizando como valores iniciais os obtidos na análise anterior, até que o valor -2Log fixasse 2 casas após o ponto, alcançado a convergência global. As tendências genéticas foram obtidas por meio da análise de regressão do valor genético do animal sobre ano de nascimento. 


\section{Resultados e discussão}

A média de idade da vaca foi 89,53 $\pm 42,62$ meses para P240 e 89,25 $\pm 42,43$ meses para P420. As médias para P240 e P420, desvios-padrão e coeficientes de variação (CV) estimados pelo programa MTDFREML foram 200,96 $\pm 70,47 \mathrm{~kg}, 35,06 \%$ e 251,66 $\pm 70,28 \mathrm{~kg}$ e 27,93\%, respectivamente. Campêlo et al. ${ }^{(5)}$ apresentaram médias de 194,99 $\pm 32,79 \mathrm{~kg}$ e 250,04 $\pm 39,06 \mathrm{~kg}$ para P240 e P420, respectivamente, em rebanho de bovinos da raça Tabapuã, ambas as médias foram inferiores às estimadas neste estudo. Os resultados apresentaram-se inferiores aos encontrados por Cyrillo et al. ${ }^{(6)}$ que determinaram para P260, em estudos com machos da raça Nelore, média de $224,70 \mathrm{~kg}$. OsórioArce e Segura-Correa ${ }^{(7)}$, em estudos com animais Brahman e seus cruzamentos com Charoles, Simental e Pardo Suíço no México, estimaram média geral para P240 de 178,5 $\pm 0,89 \mathrm{~kg}$. Os componentes de variância e covariância e as estimativas da herdabilidade para os pesos aos 240 e 420 dias encontramse na tabela 2 .

Tabela 2. Estimativas dos componentes de co(variância), parâmetros genéticos e ambientais para peso aos 240 (P240) e peso aos 420 (P420) dias de idade para animais da raça Nelore criados na região de Goiás

\begin{tabular}{lcc}
\hline & P240 & P420 \\
\hline$\sigma^{2}{ }_{\mathrm{a}}$ & 508,26 & 743,18 \\
$\sigma^{2} \mathrm{p}$ & 3421,32 & 3072,31 \\
$\mathrm{~h}^{2}{ }_{\mathrm{a}}$ & $0,15 \pm 0,02$ & $0,24 \pm 0,00$ \\
$\mathrm{~h}^{2}{ }_{\mathrm{m}}$ & $0,06 \pm 0,02$ & - \\
$\mathrm{e}^{2}$ & $0,83 \pm 0,01$ & $0,76 \pm 0,00$ \\
\hline
\end{tabular}

$\sigma_{\mathrm{a}}^{2}$ : Variância genética aditiva; $\sigma^{2} \mathrm{p}$ : Variância fenotipica total; $h^{2} \mathrm{a}:$ Herdabilidade do efeito aditivo direto; $h^{2} \mathrm{~m}$ : Herdabilidade materna; $\mathrm{e}^{2}$ : Proporção da variância ambiental sobre a variância fenotipica.

Os animais estudados apresentaram boa variabilidade, possibilitando realizar seleção no rebanho de Goiás. A herdabilidade para a característica P240 apresentou-se inferior à estimada para P420, sendo que a última é a que poderá responder melhor à seleção, principalmente por seleção massal. Frente ao exposto, realizar uma maior pressão de seleção pode gerar maiores ganhos genéticos.

Gunski et al. ${ }^{(8)}$ estimaram, para P240 para animais Nelore, herdabilidade direta de 0,27, acima do estimado para os rebanhos de Goiás. Já Buzanskas ${ }^{(1)}$ determinou, para fêmeas da raça Canchim, 0,24 de herdabilidade direta, em consonância com o determinado para P420.

Comparando critérios de seleção para características de crescimento na raça Nelore, criados em várias regiões do Brasil, Garnero et al. ${ }^{(9)}$ encontraram herdabilidade materna para (P240) de 0,06, de acordo com o estimado neste estudo. Ribeiro et al. ${ }^{(10)}$, estudando rebanho Nelore do estado de Paraíba, estimaram herdabilidade materna de 0,36 $\pm 0,08$ para P205, o que sugere um menor foco de seleção para essa característica na região de Goiás, visto que o valor da herdabilidade obtido para a herdabilidade materna foi de baixa magnitude.

Avaliando bovinos da raça Tabapuã no Brasil, Ferraz Filho et al. ${ }^{(11)}$ apresentaram estimativas de herdabilidade aditiva materna para P205(0,10), P365(0,03) e P550(0,03) indicando que os efeitos maternos tiveram importante influência sobre o peso à desmama, mas pouco efeito sobre os pesos pós-desmama, uma vez que, nessa fase de cria, existe maior dependência dos bezerros em relação a suas mães. 
As tendências genéticas para os efeitos aditivos diretos foram altamente significativas $(\mathrm{P}<0,0001)$ para ambas as características. Os resultados apontaram um incremento anual de $0,16 \%$ e $0,20 \%$ no ganho genético direto para $\mathrm{P} 240$ e P420, respectivamente, em relação à média dos pesos ajustados, sendo 0,2744 kg para P240 e de 0,5062 kg para P420 (Figura 1).

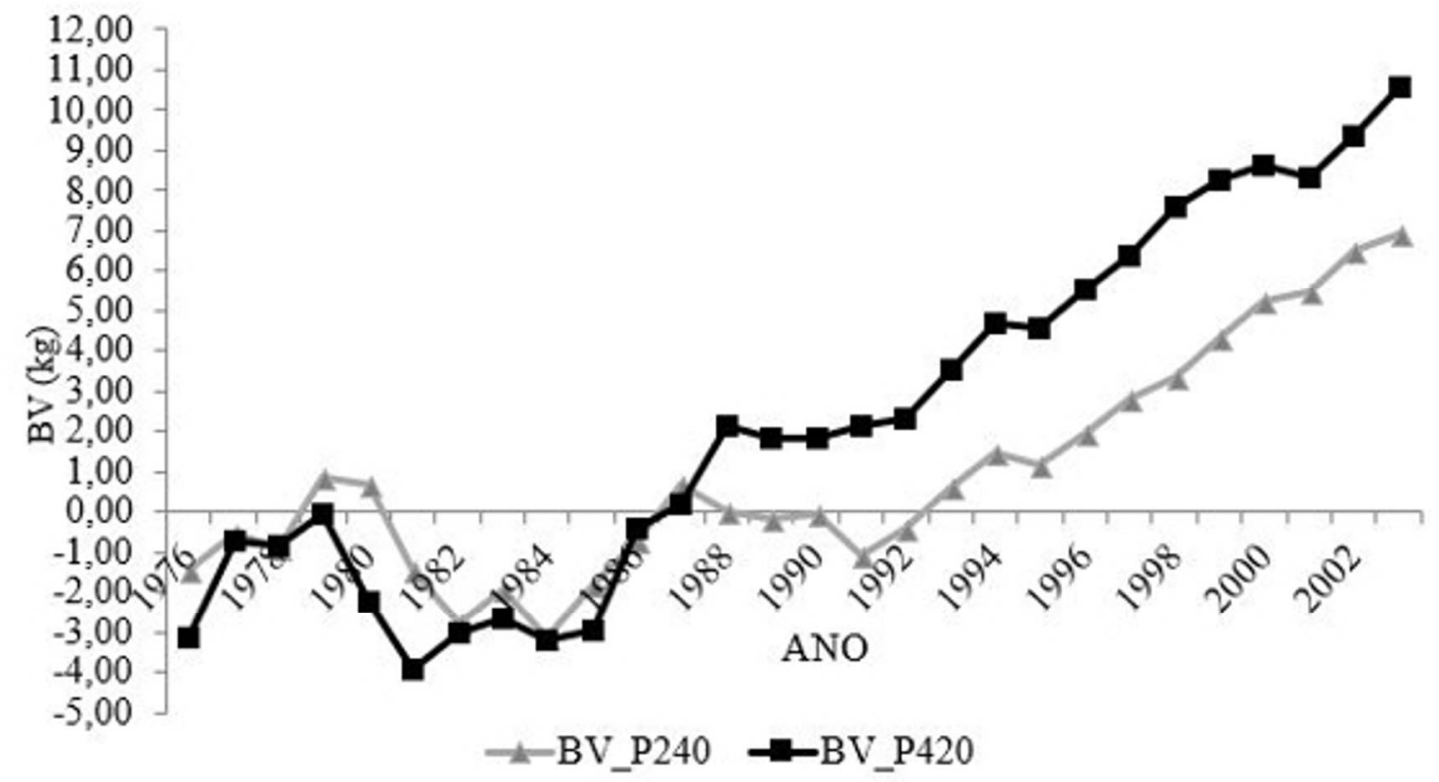

Figura 1. Tendência genética direta para valores observados e ajustados com as respectivas equações de regressão para animais da raça Nelore.

Considerando um intervalo de geração de cinco anos, o ganho médio por geração foi de $1,372 \mathrm{~kg}$ para o P240 e de 2,530 kg para P420. Já o ganho total, no período de 27 anos estudados, é de 7,410 kg e 13,660 kg para P240 e P420, respectivamente, o que demonstra uma seleção mais eficiente para o peso aos 420 dias de idade.

Ferraz Filho et al. ${ }^{(11)}$ encontraram resultados para tendências genéticas dos efeitos direto no período estudado de 0,134; 0,207, e 0,276 kg/ano, para P205, P365 e P550, respectivamente relatando também um maior ganho aos animais de idade mais avançada, porém menores do que os resultados relatados neste estudo.

Na tabela 3 pode-se observar a distribuição dos touros de acordo com seu valor genético (BV) para o estado de Goiás. Elaborou-se cinco classes com os valores das DEPS variando para a classe 1: de $=<$ -7; classe 2, variando de 2: $-7>\mathrm{bv}=<0 ; 3: 0>\mathrm{bv}=<7 ; 4: 7>$ bv $=<14$; até a classe 5: com valores genéticos bv $>14$.

Verificou-se que, dos 2.398 reprodutores que participaram da análise, 1.311 tiveram valores iguais ou inferiores a zero (54,67\%). Esses animais deveriam obrigatoriamente ser descartados de forma a implementar a produtividade do rebanho como um todo para a região. Da mesma forma, para a idade aos 420 dias, verificou-se o mesmo resultado em 53,93\% dos animais (1.294). Os resultados sugerem o uso de reprodutores das classes 3, 4 e 5, sendo os animais dessas duas últimas os que promoverão um maior ganho.

$\mathrm{Na}$ figura 2, pode se observar as duas classes abaixo de zero e as demais classes do valor genético (breed values) positivos. Dentro do possível, os animais das classes negativas devem ser identificados e eliminados dos rebanhos, evitando dessa maneira que esses continuem puxando o progresso genético para baixo. 
Tabela 3. Classes de valores genéticos [breed values (BV)], frequência (Freq.), percentagem (Percent) de touros $(n=2.398)$ por classe para os pesos aos 205 e 420 dias de idade

\begin{tabular}{ccccccccc}
\hline \multicolumn{1}{c}{ BV205 } & \multicolumn{5}{c}{ BV 420 } \\
\hline Class & N & Percent. & $\begin{array}{c}\text { Freq. } \\
\text { cumulativa }\end{array}$ & $\begin{array}{c}\text { Percent. } \\
\text { cumulativa }\end{array}$ & N & Percent. & $\begin{array}{c}\text { Freq. } \\
\text { cumulativa }\end{array}$ & $\begin{array}{c}\text { Percent. } \\
\text { cumulativa }\end{array}$ \\
\hline $\mathbf{1}$ & 226 & 9.42 & 226 & 9.42 & 347 & 14.47 & 347 & 14.47 \\
$\mathbf{2}$ & 1085 & 45.25 & 1311 & 54.67 & 947 & 39.49 & 1294 & 53.96 \\
$\mathbf{3}$ & 788 & 32.86 & 2099 & 87.53 & 658 & 27.44 & 1952 & 81.40 \\
$\mathbf{4}$ & 209 & 8.72 & 2308 & 96.25 & 253 & 10.55 & 2205 & 91.95 \\
$\mathbf{5}$ & 90 & 3.75 & 2398 & 100.00 & 193 & 8.05 & 2398 & 100.00 \\
\hline
\end{tabular}

Classes: intervalos das DEPs. $1:=\langle-7 ; 2:-7\rangle$ bv $=\langle 0 ; 3: 0\rangle$ bv $=\langle 7 ; 4: 7\rangle$ bv $=\langle 14 ; 5:$ bv $>14$;
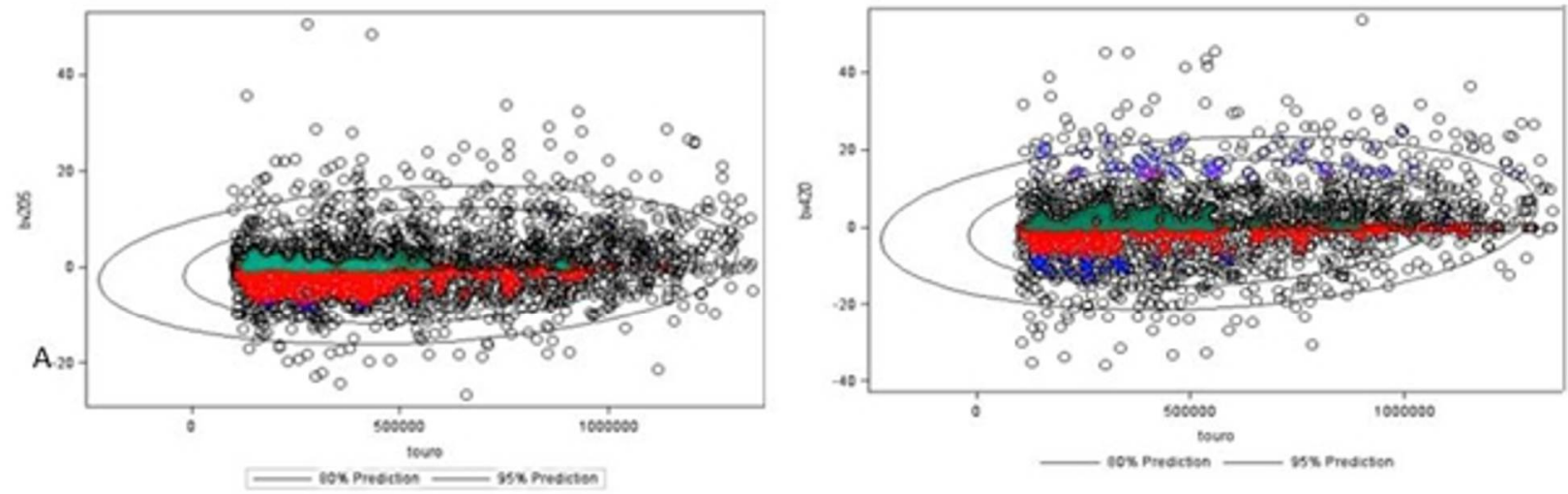

Figura 2. Distribuição dos valores genéticos de cada touro para o peso aos 205 (A) e 420 (B) dias de idade das progênies.

Usando os valores genéticos para cada progênie, realizou-se uma distribuição para intervalos de idades das vacas, considerando cada classe com intervalo de 12 meses, iniciando aos 24 meses de idade até atingir 240 meses, considerando a variável P205 dias de idade. Dessa forma, avaliou-se o valor genético das progênies de acordo com o grupo de idade da mãe, obtendo-se a equação polinomial quadrática $\mathrm{y}=-0,0082 \mathrm{x}^{2}+0,0379 \mathrm{x}+2.028$, com um $\mathrm{R}^{2}$ de 0,7922 .

A classe de novilhas entre 24 e 36 meses foi a que apresentou maior valor genético na avaliação. A classe com intervalo de 36 a 48 reduz vertiginosamente esse valor médio. Matrizes com idade acima do grupo de 144 a 156 meses mostraram que suas progênies apresentaram DEPs inferior à média (Figura 3). 


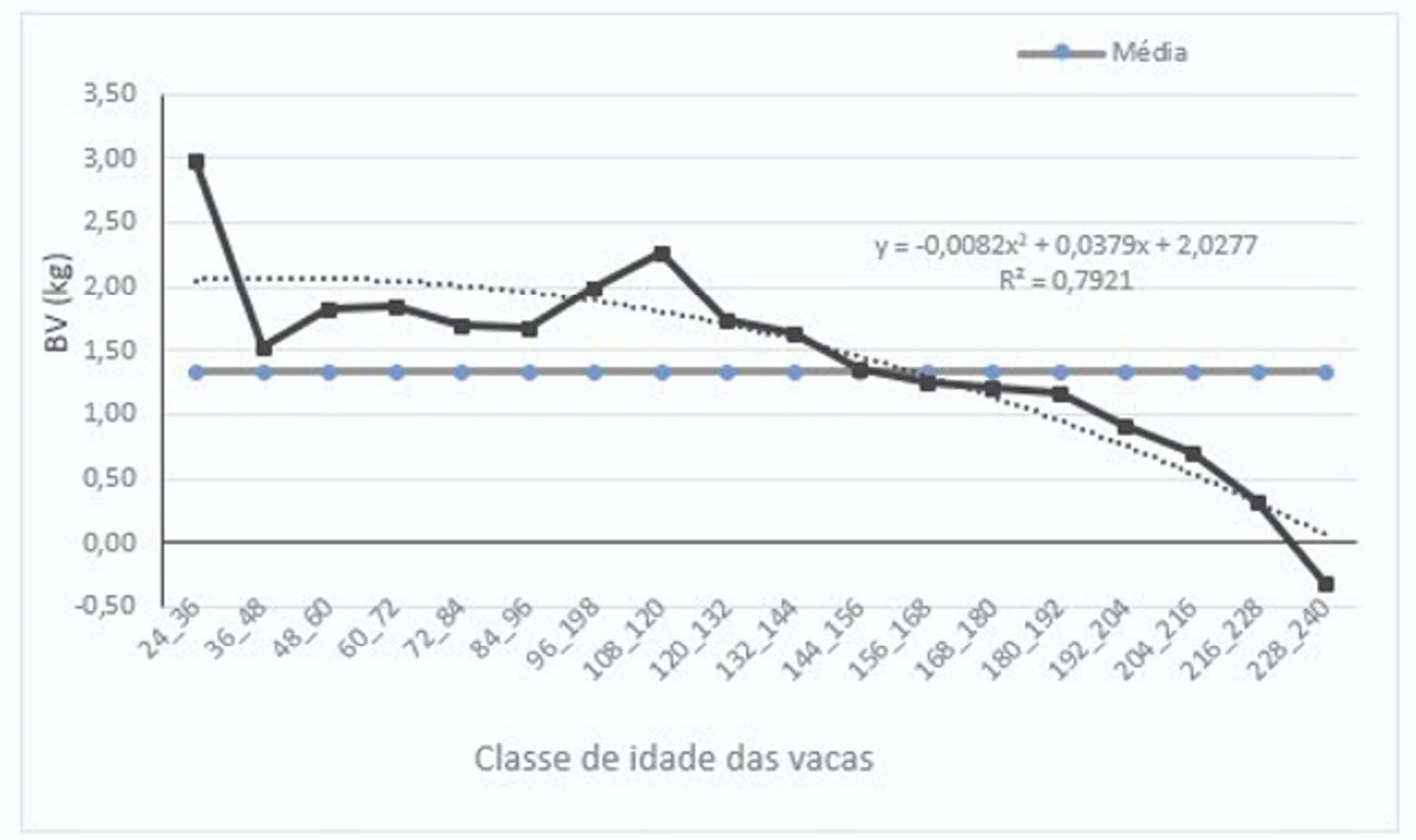

Figura 3. Valor genético para peso aos 205 dias de idade em relação à classe de idade ao parto da vaca para animais da raça Nelore.

\section{Conclusão}

A variabilidade genética e as magnitudes das herdabilidades estimadas para P240 e P420 indicam uma resposta positiva no melhoramento genético por meio de seleção, principalmente à idade de 420 dias. As tendências genéticas apresentaram ganhos anuais positivos, sendo estes voltados principalmente à característica $\mathrm{P} 420$, sugerindo uma maior pressão de seleção para peso à idade adulta na região de Goiás. Deve-se, dentro do possível, eliminar os reprodutores com valores de DEP negativos para as duas idades.

\section{Referências}

1. Buzanskas ME. Estudo da habilidade de permanência de fềmeas da raça Canchim aos 76 meses de idade. [Dissertação] Jabuticabal: Programa de Pós-Graduação em Genética e Melhoramento. Universidade Estadual Paulista; 2009, 43 p. [Acesso 2012 nov 22]. Disponível em: https://repositorio.unesp.br/bitstream/handle/11449/92590/buzanskas_me_me_jabo.pdf?sequence=1\&isAllo wed $=\mathrm{y}$

2. Gonçalves FM, Pires AV, Pereira IG, Garcia DA, Farah MM, Meira CT, CRUZ VAR. Avaliação genética para peso corporal em um rebanho Nelore. Arquivo Brasileiro de Medicina Veterinária Zootecnia, 2011; 63(1):158-164.

3. Santos GCJ. Lopes FB, Marques EG, Silva MC, Cavalcante TV, Ferreira JL. Tendência genética ara pesos padronizados aos 205, 365 e 550 dias de idades de bovinos Nelore da região norte do Brasil. Acta Scientiarum Animal Sciences, 2012; 34(1): 97-101. 
4. Arruda, ZJ., Sugai, Y. Regionalização da pecuária bovina no Brasil. Campo Grande: EMBRAPA-CNPGC; Brasília: EMBRAPA-SPI, 1994. 144 p. (EMBRAPA-CNPGC. Documentos, 58).

5. Campêlo JEG, Lopes PS, Torres RA, Silva LOC, Euclydes RF, Araujo CV, Pereira CS. Ajustamento da heterogeneidade de variâncias por transformação de escala em características de crescimento de bovinos da raça Tabapuã. Revista Ceres, 2003; 49: 481-494.

6. Cyrillo JNSG, Alencar MM, Razook AG. Modelagem e estimação de parâmetros genéticos e fenotípicos para pesos do nascimento à seleção (378 dias) de machos Nelore. Revista Brasileira de Zootecnia, Brasília, 2004; 33(6):1405-1415.

7. Osório-Arce MM, Segura-Correa JC. Estimates of breed direct, maternal and heterosis effects for weaning and yearling weights of beef cattle in the humid tropics of Mexico. Tropical and Subtropical Agroecosystems, 2010; 12: 463-469.

8. Gunski RJ, Garnero AV, Borjas AR, Bezerra LAF, Lôbo RB. Estimativas de parâmetros genéticos para características incluídas em critérios de seleção em gado Nelore. Ciência Rural; 31(4): 603-607.

9. Garnero ADV, Lôbo RB, Bezerra LAF, Oliveira HN. Comparação entre alguns critérios de seleção para crescimento na raça Nelore. Rev. Bras. Zootec., v.30, p.714-718, 2001.

10. Ribeiro MN, Pimenta Filho EC, Martins GA, Sarmento JLR, Martins Filho R. Herdabilidade para efeitos direto e materno de características de crescimento de bovinos Nelore no estado da Paraíba. Revista Brasileira de Zootecnia; 30(4):1224-1227.

11. Ferraz Filho PB, Ramos AA, Silva LOC, Souza JC, Alencar MM, Malhada CHM. Tendência genética dos efeitos direto e materno sobre os pesos à desmama e pós-desmama de bovinos da raça Tabapuã no Brasil. Revista Brasileira de Zootecnia, Brasília; 31(2): 635-640. 